\title{
Twisted Harish-Chandra sheaves and Whittaker modules: The nondegenerate case
}

\author{
Dragan Miličić and Wolfgang Soergel
}

\section{Introduction}

Let $\mathfrak{g}$ be a complex semisimple Lie algebra, $\mathscr{U}(\mathfrak{g})$ its enveloping algebra and $\mathscr{Z}(\mathfrak{g})$ the center of $\mathscr{U}(\mathfrak{g})$. Let $\mathfrak{b}$ be a fixed Borel subalgebra of $\mathfrak{g}$ and $\mathfrak{n}=[\mathfrak{b}, \mathfrak{b}]$ its nilpotent radical. A Whittaker module is a finitely generated $\mathscr{U}(\mathfrak{g})$-module which is also $\mathscr{U}(\mathfrak{n})$-finite and $\mathscr{Z}(\mathfrak{g})$-finite. The category of Whittaker modules contains as a full subcategory the category of highest weight modules, and at the other extreme, the category of nondegenerate Whittaker modules (for the precise definition see Sect. 4). In his paper [5], Kostant shows that the category of nondegenerate Whittaker modules has an extremely simple structure. The main goal of this paper is to explain Kostant's result using geometric methods - we reprove it in Sect. 5 .

Our idea was to use the localization theory of Beilinson and Bernstein [1] to transfer the study of Whittaker modules to the study of a particular category of $\mathscr{D}$ modules on the flag variety $X$ of $\mathfrak{g}$. As explained in the first four sections of the paper, our methods actually work for arbitrary Whittaker modules. The localizations of Whittaker modules are holonomic, what immediately implies that Whittaker modules are of finite length - this was proven before by McDowell [6]. He also proved that any irreducible Whittaker module is a quotient of a "standard" Whittaker module - these are a generalization of Verma modules. This leads to the natural problem of determining multiplicities of irreducible constituents of standard Whittaker modules.

Our project was started at Mathematical Sciences Research Institute in Berkeley, CA, in 1987-88, during a special year in representation theory. The first draft of

Dragan Miličić

Department of Mathematics, University of Utah, Salt Lake City, UT 84112, USA, e-mail: milicic@math.utah.edu

Wolfgang Soergel

Universität Freiburg, Mathematisches Institut, Eckerstraße 1, D-79104 Freiburg, Germany, e-mail:

wolfgang.soergel@math. uni-freiburg.de 
this paper and some of the early results on the multiplicity questions were obtained there. In particular, we realized at that time how simple is the geometric explanation of Kostant's result.

At that time, the success of the geometric approach to prove the Kazhdan-Lusztig conjectures for Verma modules was based on the fact that the localizations of highest weight modules are holonomic modules with regular singularities - this made the standard techniques used in the study of composition series questions (like the decomposition theorem) applicable. We realized immediately that the localizations of Whittaker modules have irregular singularities. Therefore, at that time, we were unable to pursue the geometric analysis of the multiplicity problem any further. Still, assuming that the decomposition theorem holds for arbitrary irreducible holonomic modules, we were able to get a number of interesting conjectural statements about the structure of the category of Whittaker modules. The most important of these statements was later proved, by completely different algebraic methods, in [10].

Recently, Mochizuki proved the decomposition theorem in full generality and made our old geometric approach rigorous [11]. Still, we decided to publish this paper in more-or-less original form to stress the simplicity of Kostant's result, deferring the general case to a future publication.

We were informed by Joseph Bernstein that he was aware that Kostant's result follows easily from localization theory.

\section{Twisted Harish-Chandra sheaves}

Let $K$ be a connected algebraic group with Lie algebra $\mathfrak{k}$ and $\phi$ a morphism of $K$ into the group of inner automorphisms $\operatorname{Int}(\mathfrak{g})$ of $\mathfrak{g}$ such that its differential induces an injection of $\mathfrak{k}$ into $\mathfrak{g}$. Hence we can identify $\mathfrak{k}$ with a subalgebra of $\mathfrak{g}$. We say that $(\mathfrak{g}, K)$ is a Harish-Chandra pair if $K$ acts by finitely many orbits on the flag variety $X$ of $\mathfrak{g}$.

Fix a Harish-Chandra pair $(\mathfrak{g}, K)$ in the following.

Let $\eta: \mathfrak{k} \longrightarrow \mathbb{C}$ be a morphism of Lie algebras, i.e., a linear form on $\mathfrak{k}$ which vanishes on $[\mathfrak{k}, \mathfrak{k}]$.

An $\eta$-twisted Harish-Chandra module is a triple $(\pi, \nu, V)$ where:

(i) $(\pi, V)$ is a finitely generated $\mathscr{U}(\mathfrak{g})$-module;

(ii) $(v, V)$ is an algebraic $K$-module;

(iii) the differential of the $K$-action on $V$ induces a $\mathscr{U}(\mathfrak{k})$-module structure on $V$ such that

$$
\pi(\xi)=v(\xi)+\eta(\xi)
$$

for any $\xi \in \mathfrak{k}$.

We denote by $\mathscr{M}_{f g}(\mathfrak{g}, K, \eta)$ the category of all $\eta$-twisted Harish-Chandra modules.

Let $\mathfrak{h}$ be the (abstract) Cartan algebra of $\mathfrak{g}[8, \S 2]$. Let $\Sigma$ be the root system in $\mathfrak{h}^{*}$ and $W$ the corresponding Weyl group. Let $\lambda \in \mathfrak{h}^{*}$ and $\theta \in W \cdot \lambda$. By a theorem of Harish-Chandra, $\theta$ determines a maximal ideal $J_{\theta}$ in $\mathscr{Z}(\mathfrak{g})$. Let $\mathscr{U}_{\theta}$ be the quotient 
of $\mathscr{U}(\mathfrak{g})$ by the ideal generated by $J_{\theta}$. Then we denote by $\mathscr{M}_{f g}\left(\mathscr{U}_{\theta}, K, \eta\right)$ the full subcategory of $\mathscr{M}_{f g}(\mathfrak{g}, K, \eta)$ consisting of modules which are actually $\mathscr{U}_{\theta}$-modules, i.e., they are annihilated by $J_{\theta}$.

In [1], Beilinson and Bernstein construct, for each $\lambda \in \mathfrak{h}^{*}$, a twisted sheaf of differential operators $\mathscr{D}_{\lambda}$ on the flag variety $X$ of $\mathfrak{g}$. For any $\lambda \in \theta$, the global sections $\Gamma\left(X, \mathscr{D}_{\lambda}\right)$ of $\mathscr{D}_{\lambda}$ are equal to $\mathscr{U}_{\theta}$.

As above, one can define the category $\mathscr{M}_{c o h}\left(\mathscr{D}_{\lambda}, K, \eta\right)$ of coherent $\mathscr{D}_{\lambda}$-modules which also admit an algebraic action of $K$. Differentiation of the $K$-action gives an action of the Lie algebra $\mathfrak{k}$, we assume that it satisfies a compatibility condition analogous to (iii) (compare [4, Appendix B], [9, Section 4]). We call the objects of $\mathscr{M}_{\text {coh }}\left(\mathscr{D}_{\lambda}, K, \eta\right) \eta$-twisted Harish-Chandra sheaves.

Clearly, the cohomology modules of $\eta$-twisted Harish-Chandra sheaves are $\eta$ twisted Harish-Chandra modules. Moreover, the localization functor $\Delta_{\lambda}$ given by $\Delta_{\lambda}(V)=\mathscr{D}_{\lambda} \otimes_{\mathscr{U}_{\theta}} V$ for a $\mathscr{U}_{\theta}$-module $V$, maps $\eta$-twisted Harish-Chandra modules into $\eta$-twisted Harish-Chandra sheaves.

Assume that $\Sigma^{+}$is the set of positive roots in $\Sigma$ such that at any point $x \in X$ it determines the nilpotent radical of the corresponding Borel subalgebra $\mathfrak{b}_{x}$. Let $\rho$ be the half-sum of roots in $\Sigma^{+}$. We say that $\lambda \in \mathfrak{h}^{*}$ is antidominant if $\alpha^{\curlyvee}(\lambda)$ is not a positive integer for any dual root $\alpha^{\swarrow}$ of $\alpha \in \Sigma^{+}$. For antidominant and regular $\lambda$ the categories $\mathscr{M}_{c o h}\left(\mathscr{D}_{\lambda}, K, \eta\right)$ and $\mathscr{M}_{f g}\left(\mathscr{U}_{\theta}, K, \eta\right)$ are equivalent $[8,3.9]$.

The next result is proved exactly as in the non-twisted case [8, 6.1].

Lemma 1.1 Any $\eta$-twisted Harish-Chandra sheaf is holonomic.

Proof. Let F $\mathscr{D}_{\lambda}$ be the natural degree filtration of $\mathscr{D}_{\lambda}$.

Let $\mathscr{V}$ be an $\eta$-twisted Harish-Chandra sheaf.

First we claim that there exists a good filtration $\left\{\mathrm{F}_{n} \mathscr{V} \mid n \in \mathbb{Z}_{+}\right\}$of $\mathscr{V}$ with the additional property that all $\mathrm{F}_{n} \mathscr{V}$ are $K$-equivariant. By twisting by a homogeneous $\mathscr{O}_{X}$-module $\mathscr{O}(v)$, for a weight $v$ in the weight lattice of $\Sigma$, we can assume that $\lambda$ is regular and antidominant. Then $V=\Gamma(X, \mathscr{V})$ is a finitely generated $\mathscr{U}_{\theta}$-module with algebraic action of $K$. This implies that it is generated by a finitedimensional $K$-invariant subspace $U$. Since by the equivalence of categories we have $\mathscr{V}=\mathscr{D}_{\lambda} \otimes_{\mathscr{U}_{\theta}} V$, the images $\mathrm{F}_{p} \mathscr{V}$ of the morphisms

$$
\mathrm{F}_{p} \mathscr{D}_{\lambda} \otimes_{\mathbb{C}} U \longrightarrow \mathscr{V}
$$

define an exhaustive $\mathscr{D}_{\lambda}$-module filtration of $\mathscr{V}$ by $K$-equivariant coherent $\mathscr{O}_{X^{-}}$ submodules. It is evident that this is a good filtration of $\mathscr{V}$.

By the $K$-equivariance of the filtration we see that $\xi \cdot \mathrm{F}_{p} \mathscr{V} \subset \mathrm{F}_{p} \mathscr{V}$ for any $\xi \in$ $\mathfrak{k} \subset \Gamma\left(X, \mathscr{D}_{\lambda}\right)$. This implies that the symbols of $\xi \in \mathfrak{k}$ annihilate $\operatorname{Gr} \mathscr{V}$. Since they vanish on the conormal bundle to any $K$-orbit in $X$, the characteristic variety $\operatorname{Ch}(\mathscr{V})$ of $\mathscr{V}$ is contained in the union of conormal bundles of $K$-orbits in $X$. Dimension of the conormal bundle to any $K$-orbit in $X$ is equal to $\operatorname{dim} X$. Since the number of orbits is finite, the dimension of the union of all such conormal bundles is also equal to $\operatorname{dim} X$. This implies that $\operatorname{dim} C h(\mathscr{V}) \leq \operatorname{dim} X$. 
In particular, this implies that twisted Harish-Chandra sheaves are of finite length. In addition we get the following consequence.

Corollary 1.2 Any $\eta$-twisted Harish-Chandra module is of finite length.

Proof. Assume that $\lambda \in \theta$ is antidominant. Then the localization $\Delta_{\lambda}(V)$ of any module $V$ in $\mathscr{M}_{f g}\left(\mathscr{U}_{\theta}, K, \eta\right)$ is in $\mathscr{M}_{c o h}\left(\mathscr{D}_{\lambda}, K, \eta\right)$. Since this Harish-Chandra sheaf is of finite length by the above remark, and $V=\Gamma\left(X, \Delta_{\lambda}(V)\right)[8,3.6]$, the assertion follows from the exactness of $\Gamma$ and the fact that global sections of an irreducible $\mathscr{D}_{\lambda}$-module are irreducible or zero [8, 3.8], [7, L.4.1].

The first example of the twisted Harish-Chandra modules was discussed in [4, Appendix B] in relation with localization theory of Harish-Chandra modules for semisimple Lie groups with infinite center.

The second example is related to Whittaker modules [5]. In this case, $K=N$. We discuss it in more details in Sect. 4.

\section{A category of $\mathfrak{n}$-finite modules}

Let $\mathscr{N}$ be the full subcategory of the category of $\mathfrak{g}$-modules consisting of modules which are

(i) finitely generated $\mathscr{U}(\mathfrak{g})$-modules;

(ii) $\mathscr{Z}(\mathfrak{g})$-finite;

(iii) $\mathscr{U}(\mathfrak{n})$-finite.

Let $\theta=W \cdot \lambda$ be a Weyl group orbit in $\mathfrak{h}^{*}$ and $J_{\theta}$ the corresponding maximal ideal in $\mathscr{Z}(\mathfrak{g})$.

Let $\mathscr{N}_{\hat{\theta}}$ be the full subcategory of $\mathscr{N}$ consisting of modules annihilated by some power of $J_{\theta}$, and $\mathscr{N}_{\theta}$ the full subcategory of $\mathscr{N}$ consisting of modules annihilated by $J_{\theta}$. Since (i) and (ii) imply that the annihilator in $\mathscr{Z}(\mathfrak{g})$ of an object in $\mathscr{N}$ is of finite codimension, we have the following result.

Lemma $2.1 \mathscr{N}=\bigoplus_{\theta \subset \mathfrak{h}^{*}} \mathscr{N}_{\hat{\theta}}$

In other words, every object in $\mathscr{N}$ is a direct sum of finitely many objects in different $\mathscr{N}_{\hat{\theta}}$.

Let $V$ be a $\mathscr{U}(\mathfrak{n})$-finite module. For $\eta \in \mathfrak{n}^{*}$ we put

$$
V_{\eta}=\left\{v \in V \mid(\xi-\eta(\xi))^{k} v=0, \xi \in \mathfrak{n}, \text { for some } k \in \mathbb{N}\right\}
$$

Then $V_{\eta} \neq 0$ implies $\eta \mid[\mathfrak{n}, \mathfrak{n}]=0$ and $V=\bigoplus_{\eta \in \mathfrak{n}^{*}} V_{\eta}$ [3, Ch. VII, $\S 1$, no. 3, Prop. 9.(i)]. If $V$ and $W$ are two $\mathscr{U}(\mathfrak{n})$-finite modules, it is easy to check that $V_{\eta} \otimes W_{\eta^{\prime}} \subset(V \otimes W)_{\eta+\eta^{\prime}}$ for any $\eta$ and $\eta^{\prime}$. Assume now that $V \in \mathscr{N}$. Since the adjoint action of $\mathfrak{n}$ on $\mathfrak{g}$ is nilpotent, we have $\mathfrak{g}=\mathfrak{g}_{0}$. Hence, we conclude that the natural map $\mathfrak{g} \otimes V \longrightarrow V$ given by $\xi \otimes v=\xi v$ maps $\mathfrak{g} \otimes V_{\eta}$ into $V_{\eta}$, i.e., $V_{\eta}$ is 
a $\mathfrak{g}$-submodule. Denote by $\mathscr{N}_{\eta}$ the full subcategory of modules with the property $V=V_{\eta}$. Then we have the following result.

Lemma 2.2 $\mathscr{N}=\bigoplus_{\eta \in \mathfrak{n}^{*}} \mathscr{N}_{\eta}$.

In other words, every object in $\mathscr{N}$ is a direct sum of finitely many objects in different $\mathscr{N}_{\eta}$. Put $\mathscr{N}_{\theta, \eta}=\mathscr{N}_{\theta} \cap \mathscr{N}_{\eta}$. Clearly, any irreducible object in $\mathscr{N}$ is in some $\mathscr{N}_{\theta, \eta}$.

Let $V$ be an object in $\mathscr{N}_{\theta, \eta}$. Then $V \otimes \mathbb{C}_{-\eta}$ is a $\mathscr{U}(\mathfrak{n})$-finite module and clearly $V \otimes \mathbb{C}_{-\eta}=\left(V \otimes \mathbb{C}_{-\eta}\right)_{0}$, i.e., for any $v \in V \otimes \mathbb{C}_{-\eta}$ we have $\mathfrak{n}^{k} \cdot v=0$ for sufficiently large $k \in \mathbb{N}$. Therefore, the $\mathfrak{n}$-action is the differential of an algebraic action of $N$ on $V \otimes \mathbb{C}_{-\eta}$. Using the natural isomorphism $V \longrightarrow V \otimes \mathbb{C}_{-\eta}$ given by $v \mapsto v \otimes 1$, we get an algebraic representation of $N$ on $V$ with differential which differs from the original action of $\mathfrak{n}$ by $\eta$, i.e., $V$ is in $\mathscr{M}_{f g}\left(\mathscr{U}_{\theta}, N, \eta\right)$. This leads us to the following result.

Lemma 2.3 $\mathscr{N}_{\theta, \eta}=\mathscr{M}_{f g}\left(\mathscr{U}_{\theta}, N, \eta\right)$.

In particular, the localization functor $\Delta_{\lambda}$ maps $\mathscr{N}_{\theta, \eta}$ into $\mathscr{M}_{c o h}\left(\mathscr{D}_{\lambda}, N, \eta\right)$. Hence, from 1.1 we deduce the following result.

Theorem 2.4 Localization $\Delta_{\lambda}(V)$ of a module $V$ in $\mathscr{N}_{\theta}$ is a holonomic $\mathscr{D}_{\lambda}$-module.

In particular, localizations are $\mathscr{D}_{\lambda}$-modules of finite length. This has the following consequence originally proved in [6].

Theorem 2.5 Any module in $\mathscr{N}$ is of finite length.

Proof. By 2.1 we can assume that $V$ is in $\mathscr{N}_{\hat{\theta}}$. Moreover, such $V$ has a finite filtration FV such that $\operatorname{Gr} V$ is in $\mathscr{N}_{\theta}$. This reduces the proof to the case of $V \in \mathscr{N}_{\theta}$. Let $V \in \mathscr{N}_{\theta}$. By 1.2 and 2.3, we see that such $V$ is of finite length.

\section{Classification of irreducible twisted Harish-Chandra sheaves}

Again, this is a simple variant of the results in the non-twisted case $[8, \S 6]$. Let $\mathscr{V}$ be an irreducible object in $\mathscr{M}_{f g}\left(\mathscr{D}_{\lambda}, K, \eta\right)$. Then its support is an irreducible $K$-invariant subvariety of $X$. Therefore, it is the closure of a $K$-orbit $Q$ in $X$. Let $i: Q \longrightarrow X$ be the natural inclusion. Then $i$ is an affine immersion. The twisted sheaf of differential operators $\mathscr{D}_{\lambda}$ on $X$ induces the twisted sheaf of differential operators $\left(\mathscr{D}_{\lambda}\right)^{i}=\mathscr{D}_{Q, \mu}$ on $Q$ where $\mu$ is the restriction of the specialization of $\lambda+\rho$ to $\mathfrak{k} \cap \mathfrak{b}_{x}$ [4, Appendix A]. By Kashiwara's equivalence of categories, the inverse image $i !(\mathscr{V})$ is an irreducible holonomic $\mathscr{D}_{Q, \mu}$-module [8, $\left.\S 4\right]$. By the compatibility condition (iii), it is also a $K$-homogeneous $\mathscr{O}_{Q}$-module such that the differential of the $K$ action differs from the action of $\mathfrak{k}$ through $\mathscr{D}_{Q, \mu}$ by $\eta$. Since $i^{!}(\mathscr{V})$ is holonomic, $i^{!}(\mathscr{V})$ is a connection on a dense open subset of $Q$ and therefore a coherent $\mathscr{O}$ module there. Since it is also $K$-equivariant, it must be coherent everywhere on $Q$, 
hence it is a connection on $Q$. We put $\tau=i^{!}(\mathscr{V})$ and denote by $\mathscr{I}(Q, \tau)$ the direct image of $\tau$ in $\mathscr{M}\left(\mathscr{D}_{\lambda}, K, \eta\right)$. The module $\mathscr{I}(Q, \tau)$ is called the standard HarishChandra sheaf attached to $(Q, \tau)$. The standard Harish-Chandra sheaf $\mathscr{I}(Q, \tau)$ has a unique irreducible Harish-Chandra subsheaf $\mathscr{L}(Q, \tau)$. The module $\mathscr{L}(Q, \tau)$ is isomorphic to $\mathscr{V}$.

Moreover, the quotient $\mathscr{I}(Q, \tau) / \mathscr{L}(Q, \tau)$ is a Harish-Chandra sheaf supported in the boundary of the closure of the $K$-orbit $Q$.

It remains to describe all $\eta$-twisted irreducible $\mathscr{D}_{Q, \mu}$-connections $\tau$ on the $K$ orbit $Q$. Let $x \in Q$. Let $B_{x}$ be the Borel subgroup of $\operatorname{Int}(\mathfrak{g})$ with the Lie algebra

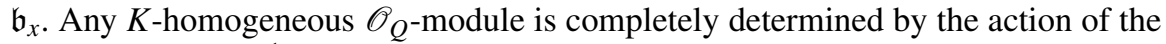
stabilizer $S_{x}=\phi^{-1}\left(\phi(K) \cap B_{x}\right)$ in the geometric fiber at $x$. By the compatibility, the irreducibility of $\tau$ implies also that it is irreducible as a $K$-homogeneous $\mathscr{O}_{Q^{-}}$ module. Hence, the representation of $S_{x}$ in the geometric fiber of $\tau$ is irreducible. Moreover, its differential is a direct sum of a number of copies of the linear form $\mu-\eta \mid\left(\mathfrak{k} \cap \mathfrak{b}_{x}\right)$ on $\mathfrak{k} \cap \mathfrak{b}_{x}$.

\section{Whittaker modules}

Let $K=N$. Let $\mathfrak{b}$ be the unique Borel subalgebra of $\mathfrak{g}$ containing $\mathfrak{n}$. Then $N$-orbits are the Bruhat cells $C(w), w \in W$, with respect to $\mathfrak{b}$, i.e., each cell $C(w)$ consists of all Borel subalgebras in relative position $w$ with respect to $\mathfrak{b}$. Let $\mathfrak{b}_{w}$ be one of such Borel subalgebras in $C(w)$. Fix a Cartan subalgebra $\mathfrak{c}$ of $\mathfrak{g}$ contained in $\mathfrak{b} \cap \mathfrak{b}_{w}$. Let $R$ be the root system of $(\mathfrak{g}, \mathfrak{c})$ and $R^{+}$the set of positive roots determined by $\mathfrak{n}$. Denote by $s: \mathfrak{h}^{*} \longrightarrow \mathfrak{c}^{*}$ the specialization determined by $\mathfrak{b}[8, \S 2]$. Then $\mathfrak{n}_{w}=\left[\mathfrak{b}_{w}, \mathfrak{b}_{w}\right]$ is spanned by the root subspaces corresponding to the roots in $s^{-1}\left(w\left(\Sigma^{+}\right)\right)$.

Now we want to discuss the compatibility condition from the end of the last section in this special case. Assume that a Bruhat cell $C(w)$ admits an irreducible $N$-homogeneous connection. First, $\mathfrak{n} \cap \mathfrak{b}_{w} \subset \mathfrak{n}_{w}$, hence we have $\mu=0$. Also, since the stabilizer of $\mathfrak{b}_{w}$ in $N$ is unipotent, the only irreducible $N$-homogeneous $\mathscr{O}_{C(w)^{-}}$ module on $C(w)$ is $\mathscr{O}_{C(w)}$. Therefore, a connection with the properties described in Sect. 3 exists on $C(w)$ if and only if $\eta \mid\left(\mathfrak{n} \cap \mathfrak{n}_{w}\right)=0$. Moreover, it is isomorphic to $\mathscr{O}_{C(w)}$. By abuse of the notation, for $\alpha \in \Sigma$ we denote by $\mathfrak{g}_{\alpha}$ the root subspace in $\mathfrak{g}$ corresponding to the root $s^{-1} \alpha \in R$. Then, the subalgebra $\mathfrak{n} \cap \mathfrak{n}_{w}$ is spanned by the root subspaces $\mathfrak{g}_{\alpha}$ for $\alpha \in \Sigma^{+} \cap w\left(\Sigma^{+}\right)$. Hence, $\eta \mid\left(\mathfrak{n} \cap \mathfrak{n}_{w}\right)=0$ if and only if $\eta \mid \mathfrak{g}_{\alpha}=0$ for $\alpha \in \Sigma^{+} \cap w\left(\Sigma^{+}\right)$.

Let $\Pi$ be the set of simple roots in $\Sigma$ corresponding to $\Sigma^{+}$. The root subspaces $\mathfrak{g}_{\alpha}, \alpha \in \Pi$, span a complement of $[\mathfrak{n}, \mathfrak{n}]$ in $\mathfrak{n}$. Therefore, $\eta \mid\left(\mathfrak{n} \cap \mathfrak{n}_{w}\right)=0$ if and only if $\eta \mid \mathfrak{g}_{\alpha}=0$ for $\alpha \in \Pi \cap w\left(\Sigma^{+}\right)$.

Let $\ell: W \longrightarrow \mathbb{Z}_{+}$be the length function on $W$ with respect to the reflections $s_{\alpha}$, $\alpha \in \Pi$. Then, for any $w \in W$, we have $\ell(w)=\operatorname{dim} C(w)$.

Let $\Theta \subset \Pi$, and let $W_{\Theta}$ be the subgroup of $W$ generated by the reflections with respect to $\alpha \in \Theta$. The set of simple roots $\Theta$ determines also a standard parabolic subalgebra $\mathfrak{p}_{\Theta}$ containing $\mathfrak{b}$. Let $P_{\Theta}$ be the corresponding parabolic subgroup in 
$\operatorname{Int}(\mathfrak{g})$. Any $P_{\Theta}$-orbit in $X$ is a disjoint union of Bruhat cells $C(t v), t \in W_{\Theta}$, for some $v \in W$. In this way, we obtain a bijection between the $P_{\Theta}$-orbits in $X$ and right $W_{\Theta}$ cosets of $W$.

The following result is well known.

Lemma 4.1 The following conditions are equivalent:

(i) $\Theta \cap w\left(\Sigma^{+}\right)=\emptyset$;

(ii) $C(w)$ is the Bruhat cell open in one of the $P_{\Theta}$-orbits in $X$;

(iii) $w$ is the longest element in one of the right $W_{\Theta}$-cosets of $W$.

Proof. By the above discussion, (ii) and (iii) are equivalent. have

(ii) $\Rightarrow$ (i) Fix a $P_{\Theta}$-orbit $O$ and let $C(w)$ be the Bruhat cell open in $O$. Then we

$$
\operatorname{dim} O=\operatorname{dim} C(w)=\ell(w)>\ell\left(s_{\alpha} w\right), \text { for any } \alpha \in \Theta .
$$

Since $\ell(v)=\operatorname{Card}\left(\Sigma^{+} \cap\left(-v\left(\Sigma^{+}\right)\right)\right)$for any $v \in W[3$, Ch. VI, $\S 1$, no. 6, Cor. 2 of Prop. 17], this means that

$$
\operatorname{Card}\left(s_{\alpha} \Sigma^{+} \cap\left(-w\left(\Sigma^{+}\right)\right)\right)=\operatorname{Card}\left(\Sigma^{+} \cap\left(-s_{\alpha} w\left(\Sigma^{+}\right)\right)\right)<\operatorname{Card}\left(\Sigma^{+} \cap\left(-w\left(\Sigma^{+}\right)\right)\right),
$$

for all $\alpha \in \Theta$. Since $s_{\alpha}$ permutes all roots in $\Sigma^{+}-\{\alpha\}$, it follows that $\alpha \notin w\left(\Sigma^{+}\right)$ for all $\alpha \in \Theta$ and $w$ satisfies (i).

(i) $\Rightarrow$ (ii) Let $\Sigma_{\Theta}$ be the root subsystem of $\Sigma$ generated by $\Theta$. Let $T$ be the set of roots in $\Sigma^{+}$which are not in $\Sigma_{\Theta}$. Since for any $\alpha \in \Theta, s_{\alpha}$ permutes the positive roots in $\Sigma^{+}-\{\alpha\}$, it follows that $s_{\alpha}(T) \subset \Sigma^{+}$. On the other hand, $s_{\alpha}$ also permutes roots in the complement of $\Sigma_{\Theta}$, i.e., $s_{\alpha}(T) \cap \Sigma_{\Theta}=\emptyset$. Therefore, $s_{\alpha}(T)=T$. Since $W_{\Theta}$ is generated by the reflections with respect to $\Theta$, we see that $T$ is $W_{\Theta}$-invariant.

Assume that $w \in W$ satisfies (i). Then $S=\Sigma^{+} \cap w\left(\Sigma^{+}\right)$is disjoint from $\Theta$. We claim that $\Sigma_{\Theta} \cap S=\emptyset$. Assume that $\beta \in \Sigma_{\Theta} \cap S$. Then $\beta \in \Sigma^{+}$, and it must be a sum of roots from $\Theta$. But $\Theta \subset-w\left(\Sigma^{+}\right)$, hence $\beta \in-w\left(\Sigma^{+}\right)$and we have a contradiction. It follows that $S \subset T$. Hence, $t(S) \subset T \subset \Sigma^{+}$for any $t \in W_{\Theta}$. In particular, for any $t \in W_{\Theta}$, we have $t(S) \subset \Sigma^{+} \cap t w\left(\Sigma^{+}\right)$. It follows that

$$
\begin{aligned}
\ell(t w)=\operatorname{Card}\left(\Sigma^{+} \cap\left(-t w\left(\Sigma^{+}\right)\right)\right)=\operatorname{Card}\left(\Sigma^{+}\right) & -\operatorname{Card}\left(\Sigma^{+} \cap t w\left(\Sigma^{+}\right)\right) \\
& \leq \operatorname{Card}\left(\Sigma^{+}\right)-\operatorname{Card}(S)=\ell(w)
\end{aligned}
$$

for any $t \in W_{\Theta}$, i.e., $C(w)$ is the Bruhat cell of maximal dimension among the cells contained in $O$.

Now, let

$$
\Theta=\left\{\alpha \in \Pi|\eta| \mathfrak{g}_{\alpha} \neq 0\right\}
$$

As we already remarked, a compatible irreducible connection exists on $C(w)$ if and only if $\Theta \cap w\left(\Sigma^{+}\right)=\emptyset$. By 4.1, this is true if and only if $C(w)$ is the open Bruhat cell in one of $P_{\Theta}$-orbits in $X$. This leads to the following result.

For a Bruhat cell $C(w)$ with the compatible irreducible connection $\mathscr{O}_{C(w)}$ denote by $\mathscr{I}(w, \lambda, \eta)$ the corresponding standard $\eta$-twisted Harish-Chandra sheaf and by $\mathscr{L}(w, \lambda, \eta)$ the corresponding irreducible $\eta$-twisted Harish-Chandra sheaf. 
Theorem 4.2 The irreducible objects in the category $\mathscr{M}_{\text {coh }}\left(\mathscr{D}_{\lambda}, N, \eta\right)$ are the modules $\mathscr{L}(w, \lambda, \eta)$ where $w \in W$ is such that $C(w)$ is an open Bruhat cell in a $P_{\Theta}$-orbit in $X$.

We can also show, that for an antidominant $\lambda$, the "costandard" Harish-Chandra sheaves $\mathscr{M}(w, \lambda, \eta)$, which are obtained from standard Harish-Chandra sheaves $\mathscr{I}(w, \lambda, \eta)$ by an appropriate holonomic dualization process, correspond under localization to the "standard" Whittaker modules studied in [6]. We are going to discuss this in a subsequent paper.

As we mentioned in the introduction, the objects in $\mathscr{M}_{c o h}\left(\mathscr{D}_{\lambda}, N, \eta\right)$ have irregular singularities in general. This is clearly visible from the following example.

Let $\mathfrak{g}=\mathfrak{s l}(2, \mathbb{C})$ and

$$
N=\left\{\left(\begin{array}{ll}
1 & 0 \\
x & 1
\end{array}\right) \mid x \in \mathbb{C}\right\} .
$$

The flag variety $X$ of $\mathfrak{g}$ is identified with $\mathbb{P}^{1}$. Let $x$ be a point in $X$. The Borel subalgebra $\mathfrak{b}_{x}$ in $\mathfrak{g}$ is the stabilizer of the line in $\mathbb{C}^{2}$ determined by $x$. Therefore, $\mathfrak{n}$ is the nilpotent radical of $\mathfrak{b}_{\infty}$ and $\mathbb{C} \subset \mathbb{P}^{1}$ is the open $N$-orbit in $X$. Let $\lambda=-\rho$. Then $\mathscr{D}_{-\rho}=\mathscr{D}_{X}$ is the sheaf of differential operators on $X$. The matrix

$$
\left(\begin{array}{ll}
1 & 0 \\
x & 1
\end{array}\right) \in N
$$

moves 0 into $x$. Hence it defines an isomorphism of $N$ onto $\mathbb{C}$. Also, if $\partial$ denotes differentiation with respect to $z$ considered as a vector field on $\mathbb{C}$, then

$$
\xi=\left(\begin{array}{ll}
0 & 0 \\
1 & 0
\end{array}\right) \in \mathfrak{n}
$$

corresponds to $\partial$ under the above isomorphism.

Let $\mathscr{I}_{\eta}$ be the standard $\eta$-twisted Harish-Chandra sheaf attached to the open orbit. Then the restriction of $\mathscr{I}_{\eta}$ to $\mathbb{C}$ is isomorphic to the quotient of $\mathscr{D}_{\mathbb{C}}$ by the left ideal generated by $\partial-\eta(\xi)$. If $\eta \neq 0$, this is a connection on $\mathbb{C}$ which has an irregular singularity at infinity.

\section{The nondegenerate case}

We say that $\eta$ is nondegenerate if $\eta \mid \mathfrak{g}_{\alpha} \neq 0$ for $\alpha \in \Pi$. In this case $\Theta=\Pi$ and $P_{\Theta}=$ $G$. Let $w_{0}$ be the longest element of the Weyl group $W$. By 4.2, in this case there exists only one irreducible object $\mathscr{L}_{\lambda}=\mathscr{L}\left(w_{0}, \lambda, \eta\right)$ in $\mathscr{M}_{c o h}\left(\mathscr{D}_{\lambda}, N, \eta\right)$. Since the quotient of the corresponding standard Harish-Chandra sheaf $\mathscr{I}_{\lambda}$ by $\mathscr{L}_{\lambda}$ must be supported in the complement of the big cell, it must be zero. Hence, we conclude that $\mathscr{L}_{\lambda}$ is equal to the standard Harish-Chandra sheaf $\mathscr{I}_{\lambda}$, i.e., $\mathscr{I}_{\lambda}$ is irreducible. 
Moreover, the space of global sections of $\mathscr{I}_{\lambda}$ is equal to the space $R\left(C\left(w_{0}\right)\right)$ of regular functions on the affine variety $C\left(w_{0}\right)$. Therefore, from $[8,3.8]$, we see that $\Gamma\left(X, \mathscr{I}_{\lambda}\right)$ is an irreducible Whittaker module for any antidominant $\lambda \in \mathfrak{h}^{*}$.

This also implies that in this case there exists a unique irreducible object in the category $\mathscr{N}_{\theta, \eta}$.

Now we want to describe these modules. Clearly, the function 1 on $X$ determines a global section of $\mathscr{I}_{\lambda}$ and it spans an $\mathfrak{n}$-stable subspace of $\Gamma\left(X, \mathscr{I}_{\lambda}\right)$ on which $\mathfrak{n}$ acts by $\eta$. Since $\mathscr{I}_{\lambda}$ is irreducible, this leads to an epimorphism of the coherent $\mathscr{D}_{\lambda}$-module $\mathscr{D}_{\lambda} \otimes_{\mathscr{U}(\mathfrak{n})} \mathbb{C}_{\eta}$ onto $\mathscr{I}_{\lambda}$. Clearly, with the $N$-action given by the tensor product of the natural action on $\mathscr{D}_{\lambda}$ with the trivial action on $\mathbb{C}, \mathscr{D}_{\lambda} \otimes_{\mathscr{U}(\mathfrak{n})} \mathbb{C}_{\eta}$ becomes an $\eta$-twisted Harish-Chandra sheaf. Moreover, since an orbit map from $N$ into $C\left(w_{0}\right)$ is an isomorphism, the restriction to the big cell $C\left(w_{0}\right)$ is an epimorphism of the $\mathscr{O}_{C\left(w_{0}\right)}$-module

$$
\left(\mathscr{D}_{\lambda} \otimes_{\mathscr{U}(\mathfrak{n})} \mathbb{C}_{\eta}\right) \mid C\left(w_{0}\right) \cong \mathscr{D}_{C\left(w_{0}\right)} \otimes_{\mathscr{U}(\mathfrak{n})} \mathbb{C}_{\eta} \cong \mathscr{O}_{C\left(w_{0}\right)} \otimes_{\mathbb{C}} \mathbb{C} \cong \mathscr{O}_{C\left(w_{0}\right)}
$$

onto the irreducible connection $\mathscr{I}_{\lambda} \mid C\left(w_{0}\right)$. Therefore, this is an isomorphism. It follows that the kernel of the morphism of $\mathscr{D}_{\lambda} \otimes_{\mathscr{U}(\mathfrak{n})} \mathbb{C}_{\eta}$ onto $\mathscr{I}_{\lambda}$ is supported on the complement of the big cell. But as we remarked before, the only object which can be supported there is 0 . This implies that $\mathscr{D}_{\lambda} \otimes_{\mathscr{U}(\mathfrak{n})} \mathbb{C}_{\eta}=\mathscr{I}_{\lambda}$. This finally proves the following result.

Theorem 5.1 Let $\eta \in \mathfrak{n}$ be nondegenerate and $\lambda \in \mathfrak{h}^{*}$. Then the only irreducible object in $\mathscr{M}_{\text {coh }}\left(\mathscr{D}_{\lambda}, N, \eta\right)$ is $\mathscr{D}_{\lambda} \otimes_{\mathscr{U}(\mathfrak{n})} \mathbb{C}_{\eta}$.

We also have an analogous result for $\mathscr{N}_{\theta, \eta}$. It was originally proved by Kostant in his work on Whittaker modules [5].

Theorem 5.2 Let $\eta \in \mathfrak{n}$ be nondegenerate. Then the only irreducible module in $\mathscr{N}_{\theta, \eta}$ is $\mathscr{U}_{\theta} \otimes_{\mathscr{U}(\mathfrak{n})} \mathbb{C}_{\eta}$.

Proof. Let $\lambda \in \theta$ be antidominant. Then

$$
\Delta_{\lambda}\left(\mathscr{U}_{\theta} \otimes_{\mathscr{U}(\mathfrak{n})} \mathbb{C}_{\eta}\right)=\mathscr{D}_{\lambda} \otimes_{\mathscr{U}_{\theta}}\left(\mathscr{U}_{\theta} \otimes_{\mathscr{U}(\mathfrak{n})} \mathbb{C}_{\eta}\right)=\mathscr{D}_{\lambda} \otimes_{\mathscr{U}(\mathfrak{n})} \mathbb{C}_{\eta}=\mathscr{I}_{\lambda} .
$$

Therefore, by $[8,3.6]$, we have

$$
\Gamma\left(X, \mathscr{I}_{\lambda}\right)=\mathscr{U}_{\theta} \otimes_{\mathscr{U}(\mathfrak{n})} \mathbb{C}_{\eta}
$$

and this is the unique irreducible object in $\mathscr{M}_{f g}\left(\mathscr{U}_{\theta}, N, \eta\right)$.

A vector in a Whittaker module which spans an $\mathfrak{n}$-stable subspace is called a Whittaker vector.

Corollary 5.3 All Whittaker vectors in $\mathscr{U}_{\theta} \otimes_{\mathscr{U}(\mathfrak{n})} \mathbb{C}_{\eta}$ are proportional to $1 \otimes 1$.

Proof. By the preceding argument we see that Whittaker vectors correspond exactly to $N$-invariant sections of $\mathscr{I}_{\lambda}$. These sections are exactly constant functions on the open cell $C\left(w_{0}\right)$. 
Since the global sections of $\mathscr{D}_{\lambda}$ clearly operate faithfully on global sections of $\mathscr{I}_{\lambda}$ we get the following consequence [5].

Corollary 5.4 The action of $\mathscr{U}_{\theta}$ on $\mathscr{U}_{\theta} \otimes_{\mathscr{U}(\mathfrak{n})} \mathbb{C}_{\eta}$ is faithful.

Consider now an arbitrary object $\mathscr{V}$ in $\mathscr{M}_{c o h}\left(\mathscr{D}_{\lambda}, N, \eta\right)$. Its restriction onto the big cell is an $N$-homogeneous connection. Since the stabilizer in $N$ of an arbitrary point in the big cell is trivial, this connection is equal to a sum of copies of the irreducible connection on $C\left(w_{0}\right)$. Since the restriction is left adjoint to direct image, this implies that there exists a natural morphism $\varphi$ of $\mathscr{V}$ into a sum of copies of $\mathscr{I}_{\lambda}$. By the preceding discussion, since the kernel and the cokernel of $\varphi$ are supported in the complement of the big cell, they are equal to zero. This leads to the following results which show the extreme simplicity of the categories $\mathscr{M}_{c o h}\left(\mathscr{D}_{\lambda}, N, \eta\right)$ and $\mathscr{N}_{\theta, \eta}$ for nondegenerate $\eta$.

Theorem 5.5 Let $\eta \in \mathfrak{n}$ be nondegenerate. Then all objects in $\mathscr{M}_{c o h}\left(\mathscr{D}_{\lambda}, N, \eta\right)$ are finite sums of irreducible objects $\mathscr{D}_{\lambda} \otimes_{\mathscr{U}(\mathfrak{n})} \mathbb{C}_{\eta}$.

Theorem 5.6 Let $\eta \in \mathfrak{n}$ be nondegenerate. Then modules in $\mathscr{N}_{\theta, \eta}$ are finite sums of irreducible modules $\mathscr{U}_{\theta} \otimes_{\mathscr{U}(\mathfrak{n})} \mathbb{C}_{\eta}$.

Now we want to describe the structure of the category $\mathscr{N}_{\eta}$ for a nondegenerate $\eta \in \mathfrak{n}^{*}$.

We start with a simple technical result. The enveloping algebra $\mathscr{U}(\mathfrak{g})$ has a natural structure of a left $\mathscr{Z}(\mathfrak{g}) \otimes_{\mathbb{C}} \mathscr{U}(\mathfrak{n})$-module given by left multiplication. The following generalization of a classical result of Kostant must be well known. ${ }^{1}$

Lemma $5.7 \mathscr{U}(\mathfrak{g})$ is free as a $\mathscr{Z}(\mathfrak{g}) \otimes_{\mathbb{C}} \mathscr{U}(\mathfrak{n})$-module.

Proof. Let $\left(\mathscr{U}_{p}(\mathfrak{g}) ; p \in \mathbb{Z}_{+}\right)$denote the natural filtration of the enveloping algebra $\mathscr{U}(\mathfrak{g})$ of $\mathfrak{g}$.

Fix a Cartan subalgebra $\mathfrak{c}$ and a nilpotent subalgebra $\overline{\mathfrak{n}}$ opposite to $\mathfrak{n}$. Then we have $\mathfrak{g}=\mathfrak{n} \oplus \mathfrak{c} \oplus \overline{\mathfrak{n}}$, and by the Poincaré-Birkhoff-Witt theorem it follows that $\mathscr{U}(\mathfrak{g})=\mathscr{U}(\mathfrak{n}) \otimes_{\mathbb{C}} \mathscr{U}(\mathfrak{c}) \otimes_{\mathbb{C}} \mathscr{U}(\overline{\mathfrak{n}})$ as a left $\mathscr{U}(\mathfrak{n})$-module for left multiplication. Then we define a linear space filtration $\mathrm{F} \mathscr{U}(\mathfrak{g})$ of $\mathscr{U}(\mathfrak{g})$ via

$$
\mathrm{F}_{p} \mathscr{U}(\mathfrak{g})=\mathscr{U}(\mathfrak{n}) \otimes_{\mathbb{C}} \mathscr{U}_{p}(\mathfrak{c}) \otimes_{\mathbb{C}} \mathscr{U}(\overline{\mathfrak{n}}) .
$$

Clearly, the natural filtration of $\mathscr{U}(\mathfrak{g})$ is finer than $\mathrm{F} \mathscr{U}(\mathfrak{g})$, i.e., $\mathscr{U}_{p}(\mathfrak{g}) \subset \mathrm{F}_{p} \mathscr{U}(\mathfrak{g})$ for all $p \in \mathbb{Z}_{+}$.

We define a filtration on $\mathscr{Z}(\mathfrak{g}) \otimes_{\mathbb{C}} \mathscr{U}(\mathfrak{n})$, by

$$
\mathrm{F}_{p}\left(\mathscr{Z}(\mathfrak{g}) \otimes_{\mathbb{C}} \mathscr{U}(\mathfrak{n})\right)=\left(\mathscr{U}_{p}(\mathfrak{g}) \cap \mathscr{Z}(\mathfrak{g})\right) \otimes_{\mathbb{C}} \mathscr{U}(\mathfrak{n}), p \in \mathbb{Z}_{+} .
$$

In this way, $\mathscr{Z}(\mathfrak{g}) \otimes_{\mathbb{C}} \mathscr{U}(\mathfrak{n})$ becomes a filtered ring. The corresponding graded ring $\operatorname{Gr}\left(\mathscr{Z}(\mathfrak{g}) \otimes_{\mathbb{C}} \mathscr{U}(\mathfrak{n})\right)$ is equal to $\operatorname{Gr}(\mathscr{Z}(\mathfrak{g})) \otimes_{\mathbb{C}} \mathscr{U}(\mathfrak{n})$. The Harish-Chandra homomorphism $\gamma: \mathscr{Z}(\mathfrak{g}) \longrightarrow \mathscr{U}(\mathfrak{c})$ is compatible with the natural filtrations and the homomorphism $\operatorname{Gr} \gamma$ is an isomorphism of $\operatorname{Gr} \mathscr{Z}(\mathfrak{g})$ onto the subalgebra $I(\mathfrak{c})$ of all

\footnotetext{
${ }^{1}$ One of us learned this argument to prove Kostant's result from Wilfried Schmid in 1977.
} 
$W$-invariants in $S(\mathfrak{c})$ [3, Ch. VIII, $\S 8$, no. 5]. Therefore,

$$
\operatorname{Gr}\left(\mathscr{Z}(\mathfrak{g}) \otimes_{\mathbb{C}} \mathscr{U}(\mathfrak{n})\right)=I(\mathfrak{c}) \otimes_{\mathbb{C}} \mathscr{U}(\mathfrak{n})
$$

Let $z \in \mathscr{U}_{p}(\mathfrak{g}) \cap \mathscr{Z}(\mathfrak{g})$. Then, by the definition of the Harish-Chandra homomorphism, we have $z-\gamma(z) \in \mathfrak{n} \mathscr{U}_{p-1}(\mathfrak{g})$. Hence, we have

$$
\begin{aligned}
& z \mathscr{U}_{q}(\mathfrak{c}) \subset \gamma(z) \mathscr{U}_{q}(\mathfrak{c})+\mathfrak{n} \mathscr{U}_{p-1}(\mathfrak{g}) \mathscr{U}_{q}(\mathfrak{c}) \subset \gamma(z) \mathscr{U}_{q}(\mathfrak{c})+\mathfrak{n} \mathscr{U}_{p+q-1}(\mathfrak{g}) \\
& \subset \gamma(z) \mathscr{U}_{q}(\mathfrak{c})+\mathrm{F}_{p+q-1} \mathscr{U}_{(\mathfrak{g}) \subset \mathrm{F}_{p+q}} \mathscr{U}(\mathfrak{g})
\end{aligned}
$$

for any $q \in \mathbb{Z}_{+}$. This implies first that

$$
z \mathrm{~F}_{q} \mathscr{U}(\mathfrak{g}) \subset \mathrm{F}_{p+q} \mathscr{U}(\mathfrak{g}), q \in \mathbb{Z}_{+} ;
$$

i.e., the filtration $\mathrm{F} \mathscr{U}(\mathfrak{g})$ is compatible with the action of $\mathscr{Z}(\mathfrak{g}) \otimes_{\mathbb{C}} \mathscr{U}(\mathfrak{n})$. Therefore, $\mathscr{U}(\mathfrak{g})$ is a filtered $\mathscr{Z}(\mathfrak{g}) \otimes_{\mathbb{C}} \mathscr{U}(\mathfrak{n})$-module. Moreover, the corresponding graded module is

$$
\operatorname{Gr} \mathscr{U}(\mathfrak{g})=\mathscr{U}(\mathfrak{n}) \otimes_{\mathbb{C}} S(\mathfrak{c}) \otimes_{\mathbb{C}} \mathscr{U}(\overline{\mathfrak{n}})
$$

with the obvious action of $I(\mathfrak{c}) \otimes_{\mathbb{C}} \mathscr{U}(\mathfrak{n})$. Since $S(\mathfrak{c})$ is a free $I(\mathfrak{c})$-module by [3, Ch. V, $\S 5$, no. 5, Thm. 4], it follows that $\operatorname{Gr} \mathscr{U}(\mathfrak{g})$ is a free $I(\mathfrak{c}) \otimes_{\mathbb{C}} \mathscr{U}(\mathfrak{n})$-module.

This easily implies that $\mathscr{U}(\mathfrak{g})$ is a free $\mathscr{Z}(\mathfrak{g}) \otimes_{\mathbb{C}} \mathscr{U}(\mathfrak{n})$-module [2, Ch. III, $\S 2$, no. 8, Cor. 3. of Thm. 1].

Let $U$ be a finite dimensional $\mathscr{Z}(\mathfrak{g})$-module. Consider it as a $\mathscr{Z}(\mathfrak{g}) \otimes_{\mathbb{C}} \mathscr{U}(\mathfrak{n})$ module, where $\mathfrak{n}$ acts by multiplication by $\eta$. Let

$$
I_{\eta}(U)=\mathscr{U}(\mathfrak{g}) \otimes_{\mathscr{Z}}(\mathfrak{g}) \otimes_{\mathbb{C}} \mathscr{U}(\mathfrak{n})
$$

we consider it as a $\mathscr{U}(\mathfrak{g})$-module by left multiplication in the first factor. By the preceding lemma, the functor $I_{\eta}$ from the category of finite-dimensional $\mathscr{Z}(\mathfrak{g})$ modules into the category of $\mathscr{U}(\mathfrak{g})$-modules is exact. It maps finite-dimensional $\mathscr{Z}(\mathfrak{g})$-modules into $\mathscr{Z}(\mathfrak{g})$-finite, finitely generated $\mathscr{U}(\mathfrak{g})$-modules. Moreover, since the action of $\mathfrak{n}$ on $I_{\eta}(U)$ is the quotient of action on the tensor product $\mathscr{U}(\mathfrak{g}) \otimes_{\mathbb{C}} U$ where $\mathfrak{n}$ acts on the first factor by the adjoint action, we see immediately that $I_{\eta}(U)$ is $\mathscr{U}(\mathfrak{n})$-finite. Hence, it is a Whittaker module. In addition, since the action of $\mathfrak{n}$ on $\mathscr{U}(\mathfrak{g})$ is nilpotent, we conclude that $I_{\eta}(U)$ is in $\mathscr{N}_{\eta}$. Therefore $I_{\eta}$ is an exact functor from the category of finite-dimensional $\mathscr{Z}(\mathfrak{g})$-modules into the category $\mathscr{N}_{\eta}$.

Assume that $\operatorname{dim} U=1$. Then a maximal ideal in $\mathscr{Z}(\mathfrak{g})$ annihilates $U$. Hence, we see that $I_{\eta}(U)=\mathscr{U}_{\theta} \otimes_{\mathscr{U}(\mathfrak{n})} \mathbb{C}_{\eta}$ for some Weyl group orbit $\theta$ in $\mathfrak{h}^{*}$. Moreover, $I_{\eta}(U)$ is irreducible by 5.2 .

By the exactness of the functor $I_{\eta}$, we immediately conclude that

$$
\text { length } I_{\eta}(U)=\operatorname{dim} U
$$

for any finite-dimensional $\mathscr{Z}(\mathfrak{g})$-module $U$. 
On the other hand, for a Whittaker module $V$ in $\mathscr{N}_{\eta}$, let $\mathrm{Wh}(V)$ denote the space of all Whittaker vectors. Clearly, $\mathrm{Wh}(V)$ is $\mathscr{Z}(\mathfrak{g})$-invariant.

Lemma 5.8 The functor Wh from the category $\mathscr{N}_{\eta}$ into the category of $\mathscr{Z}(\mathfrak{g})$ modules is exact.

Proof. The subspace $\mathrm{Wh}(V)$ of Whittaker vectors in $V$ can be identified with the module of n-invariants of $V$ with respect to the $V$-action. Therefore, it is enough to prove that $H^{1}(\mathfrak{n}, V)=0$ for any Whittaker module $V$, where the cohomology is calculated with respect to the $v$-action.

Consider first the case of irreducible Whittaker modules. As we remarked before an irreducible Whittaker module (with $v$-action) is isomorphic to the algebra of regular functions $R\left(C\left(w_{0}\right)\right)$ on open cell $C\left(w_{0}\right)$ with the natural action of $N$. Since an orbit map is an isomorphism of $N$ onto the open cell, it is enough to know that groups $H^{i}(\mathfrak{n}, R(N))=0$ for $i \geq 1$. This is a well-known fact (compare [9, Lemma 1.9], for example).

Consider now an arbitrary Whittaker module $V$. Let $V^{\prime}$ be an irreducible submodule of $V$ and consider the exact sequence

$$
0 \longrightarrow V^{\prime} \longrightarrow V \longrightarrow V^{\prime \prime} \longrightarrow 0 .
$$

From the long exact sequence of Lie algebra cohomology, we see that $H^{i}(\mathfrak{n}, V)=$ $H^{i}\left(\mathfrak{n}, V^{\prime \prime}\right)$ for $i \geq 1$. Hence, by induction on the length of $V$, we conclude that $H^{i}(\mathfrak{n}, V)=0$ for $i \geq 1$.

By 5.3, we see that for any irreducible Whittaker module in $\mathscr{N}_{\eta}$, the vector space $\mathrm{Wh}(V)$ is one-dimensional. Therefore, by induction on the length of Whittaker modules and using the exactness of Wh, we immediately get

$$
\operatorname{dim} \operatorname{Wh}(V)=\text { length }(V)
$$

for any $V$ in $\mathscr{N}_{\eta}$. In particular, $\mathrm{Wh}(V)$ is finite-dimensional. Therefore, $\mathrm{Wh}$ is an exact functor from the category $\mathscr{N}_{\eta}$ into the category of finite-dimensional $\mathscr{Z}(\mathfrak{g})$ modules. It is easy to check that

$$
\operatorname{Hom}_{\mathscr{U}(\mathfrak{g})}\left(I_{\eta}(U), V\right)=\operatorname{Hom}_{\mathscr{Z}(\mathfrak{g})}(U, \operatorname{Wh}(V)),
$$

i.e., the functor $\mathrm{Wh}$ is the right adjoint of $I_{\eta}$.

Clearly, the linear map $u \longmapsto 1 \otimes u$ from $U$ into $I_{\eta}(U)$ is injective, and its image is in $\mathrm{Wh}\left(I_{\eta}(U)\right)$. Since

$$
\operatorname{dim} \operatorname{Wh}\left(I_{\eta}(U)\right)=\text { length } I_{\eta}(U)=\operatorname{dim} U,
$$

it follows that the adjointness morphism $U \longrightarrow \mathrm{Wh}\left(I_{\eta}(U)\right)$ is an isomorphism. Conversely, let $V$ be a Whittaker module in $\mathscr{N}_{\eta}$. Then we have the adjointness morphism $I_{\eta}(\mathrm{Wh}(V)) \longrightarrow V$. Let $K$ be its kernel and $C$ the cokernel. Then we have the exact sequence 


$$
0 \longrightarrow \mathrm{Wh}(K) \longrightarrow \mathrm{Wh}\left(I_{\eta}(\mathrm{Wh}(V))\right) \longrightarrow \mathrm{Wh}(V) \longrightarrow \mathrm{Wh}(C) \longrightarrow 0
$$

and by the preceding remark, the third arrow is an isomorphism. Hence, $\mathrm{Wh}(K)=0$ and $\mathrm{Wh}(C)=0$, i.e., $K=C=0$ and $I_{\eta}(\mathrm{Wh}(V)) \longrightarrow V$ is an isomorphism. Therefore, we established the following result.

Theorem 5.9 Let $\eta \in \mathfrak{n}^{*}$ be nondegenerate. Then the functor $I_{\eta}$ is an equivalence of the category of finite dimensional $\mathscr{Z}(\mathfrak{g})$-modules with $\mathscr{N}_{\eta}$. Its quasi-inverse is the functor Wh.

\section{References}

1. Alexander Beŭlinson and Joseph Bernstein, Localisation de $\mathfrak{g}$-modules, C. R. Acad. Sci. Paris Sér. I Math. 292 (1981), no. 1, 15-18.

2. Nicolas Bourbaki, Algèbre commutative, Mason, Paris.

3. __ Groupes et algèbres de Lie, Mason, Paris.

4. Henryk Hecht, Dragan Miličić, Wilfried Schmid, and Joseph A. Wolf, Localization and standard modules for real semisimple Lie groups. I. The duality theorem, Invent. Math. 90 (1987), no. 2, 297-332.

5. Bertram Kostant, On Whittaker vectors and representation theory, Invent. Math. 48 (1978), 101-184.

6. Edward McDowell, On modules induced from Whittaker modules, J. Algebra 96 (1985), no. 1, 161-177.

7. Dragan Miličić, Localization and representation theory of reductive Lie groups, unpublished manuscript available at http://math. utah.edu/ milicic.

8. __ Algebraic $\mathscr{D}$-modules and representation theory of semisimple Lie groups, The Penrose transform and analytic cohomology in representation theory (South Hadley, MA, 1992), Amer. Math. Soc., Providence, RI, 1993, pp. 133-168.

9. Dragan Miličić and Pavle Pandžić, Equivariant derived categories, Zuckerman functors and localization, Geometry and representation theory of real and $p$-adic groups (Córdoba, 1995), Progr. Math., vol. 158, Birkhäuser Boston, Boston, MA, 1998, pp. 209-242.

10. Dragan Miličić and Wolfgang Soergel, The composition series of modules induced from Whittaker modules, Comment. Math. Helv. 72 (1997), no. 4, 503-520.

11. Takuro Mochizuki, Wild harmonic bundles and wild pure twistor D-modules, Astérisque (2011), no. 340 . 